\title{
Spin-wave Instability Theory for Ferromagnetic Nanostructures
}

\author{
Z. Haghshenasfard, H.T. Nguyen, M.G. Cottam* \\ Department of Physics and Astronomy, University of Western Ontario London, Ontario N6A 3K7, Canada
}

\begin{abstract}
A microscopic, or Hamiltonian-based, theory is outlined for studying the spin-wave instability thresholds of the parametric processes occurring in ferromagnetic nanostructures under conditions of pumping with a microwave field. Most previous work has concentrated on spheres or films with dimensions of order several $\mu \mathrm{m}$ or more, with the theoretical interpretation being made in terms of macroscopic (or continuum) methods. At smaller length scales, as in ultrathin films and nanowires with thickness or lateral dimensions less than about $100 \mathrm{~nm}$, the discreteness of the quantized spin waves and their spatial distributions become modified, making it more appropriate to employ a microscopic approach to the nonlinear dynamics with a lattice of effective spins interacting through the magnetic dipole-dipole and exchange interactions. Effects of microwave pumping (in either the parallel or perpendicular configuration) are incorporated in calculations for the instability thresholds of the quantized spin-wave bands in different nanostructures and materials.
\end{abstract}

DOI: $10.12693 /$ APhysPolA.127.192

PACS: 75.30.Ds, 75.75.-c, 75.78.-n

\section{Introduction}

The magnetization dynamics of ordered magnetic nanostructures (such as films, wires, rings, etc., and their arrays) have generated much attention in recent years as a result of advances in materials growth techniques and the study of surfaces and interfaces. In particular, there has been renewed interest in spin-wave (SW) parametric processes in such structures (see, e.g., [1-3]).

Following the pioneering work on SW instabilities in ferromagnetic systems under microwave pumping by Suhl [4], Schlomann [5], and others, there has been an extensive amount of experimental and theoretical effort devoted to this field (for reviews see, e.g., [6-11]). Until recently, most of these studies applied to either unbounded samples or to spheres and films with dimensions of typically several $\mathrm{mm}$ or $\mu \mathrm{m}$ for which macroscopic (or continuum) descriptions are generally applicable. In finite samples boundary effects need to be taken into account, resulting in spatial quantization of the SW modes. In turn, there is a modification of the SW instability thresholds and additional selection rules under microwave pumping (see [12-15]).

The focus of our present work is a theoretical study of $\mathrm{SW}$ instability processes in structures where at least one of the sample dimensions is of the order $100 \mathrm{~nm}$ or less. The quantization of the SWs is much more pronounced and surface effects become more dominant. Moreover, it becomes more appropriate to use a microscopic (or Hamiltonian-based) method with a discrete lattice of effective spins interacting through exchange and dipoledipole terms, rather than a macroscopic continuum ap-

${ }^{*}$ corresponding author; e-mail: cottam@uwo.ca proach. Very recently we have utilized such an approach to consider the special case of parallel pumping in ultrathin magnetic films [16]. In the present work we are motivated to extend the calculations to magnetic nanowires (which include stripes and ribbons) to compare these lowdimensional systems and also to consider the case of perpendicular pumping. The theoretical techniques are generalizations, in order to include the microwave pumping fields, of earlier work on films [17, 18], wires [19-21] and magnonic crystals [22].

The calculations for the SW instability thresholds are outlined in Sect. 2, where we highlight effects of the different dimensionality (in terms of wave vectors and translational symmetry) for the films and wire cases. Then numerical examples are given in Sect. 3 for different materials to illustrate the two geometries and the crucial role of dipole-dipole to exchange ratio for interactions. Finally the conclusions are given in Sect. 4 .

\section{Theoretical method}

The two ferromagnetic nanostructures considered here are ultrathin films and nanowires (or stripes) with a rectangular cross-section. They have, respectively, two and one directions of translational symmetry and are depicted in Fig. 1. The ultrathin film with (010) surfaces is modelled as having $N$ atomic layers of spins arranged, for simplicity, on a simple cubic (sc) lattice structure with lattice constant $a$, while the nanowire (stripe) is modelled as having a rectangular cross section of $N_{x} \times N_{y}=N$ atomic spins (in the $x y$ plane). Typically, for both structures the preferred direction of spin ordering corresponds to the static magnetization along the $z$ direction, unless changed by the applied static and microwave fields.

The total spin Hamiltonian for each structure can be written in a common form as 


$$
\begin{aligned}
H & =-\frac{1}{2} \sum_{i n, j m} J_{i n, j m} S_{i n} \cdot S_{j m} \\
& +\frac{1}{2} g^{2} \mu_{\mathrm{B}}^{2} \sum_{i n, j m} \sum_{\alpha, \beta} D_{i n, j m}^{\alpha, \beta} S_{i n}^{\alpha} S_{j m}^{\beta} \\
& -g \mu_{\mathrm{B}} \sum_{i n} H_{0} \cdot S_{i n}+H_{\mathrm{p}},
\end{aligned}
$$

where the indices $i$ and $j$ refer to the spin sites within any atomic layer (in the film case) or to those sites along the $z$-directed lines of spins (in the nanowire case), while $n$ and $m(=1,2, \ldots, N)$ label the layers of the film in the $y$ direction or the spin sites in any cross-section of the nanowire. The first term in the Hamiltonian represents the short-range exchange interactions $J_{i n, j m}$ acting between the spins $\boldsymbol{S}_{i n}$ and $\boldsymbol{S}_{j m}$ at a distance apart specified by $\boldsymbol{r}_{i n, j m}$. The second term describes the long-range dipole-dipole interactions between spins with the components of the coupling tensor given by

$$
\boldsymbol{D}_{i n, j m}^{\alpha, \beta}=\frac{\left|\boldsymbol{r}_{i n, j m}\right|^{2} \delta_{\alpha, \beta}-3 \boldsymbol{r}_{i n, j m}^{\alpha} \boldsymbol{r}_{i n, j m}^{\beta}}{\left|\boldsymbol{r}_{i n, j m}\right|^{5}},
$$

where $\alpha, \beta=x, y, z$, and with $g$ and $\mu_{\mathrm{B}}$ denoting the Landé $g$-factor and Bohr magneton, respectively. The next term is the Zeeman energy due to a static applied magnetic field $\boldsymbol{H}_{0}$, which usually we take to be along the $z$ direction. Finally, $H_{\mathrm{p}}$ is the interaction Hamiltonian corresponding to the microwave pumping magnetic field $\boldsymbol{h}_{0}$ written as:

$$
H_{\mathrm{p}}=-g \mu_{\mathrm{B}} \exp \left(-\mathrm{i} \omega_{\mathrm{p}} t\right) \sum_{i n} h_{0} \cdot S_{i n} .
$$

Here we have taken a time dependence corresponding to a Fourier component with angular frequency $\omega_{\mathrm{p}}$.

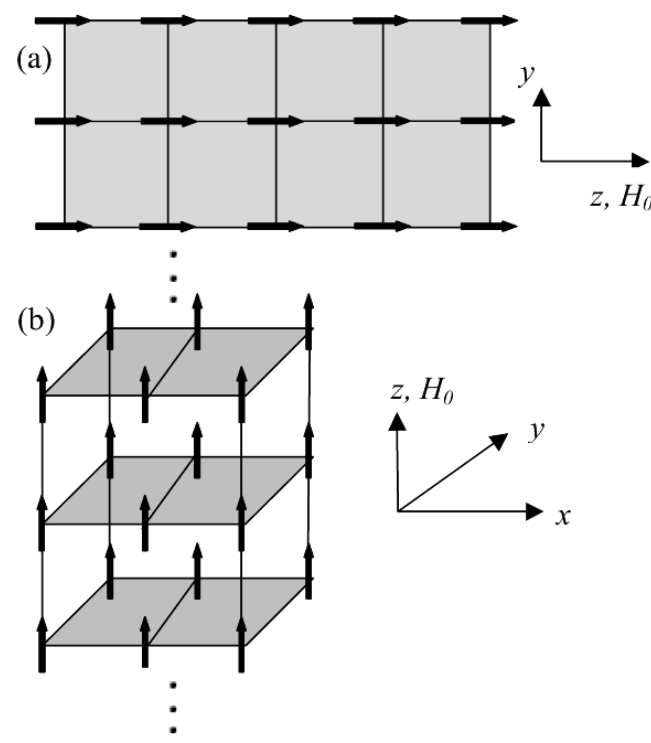

Fig. 1. Assumed nanostructure geometries and choices of coordinate axes: $(a)$ an ultrathin film with translational symmetry in the $x z$ plane and finite thickness in the $y$ direction (illustrated for $N=3$ layers); $(b)$ a rectangular nanowire with translational symmetry along the $z$ direction and finite in the $x y$ plane (illustrated for $N=3 \times 2=6$ lines of spins).
In order to study SWs and their instability properties at low temperatures $T \ll T_{\mathrm{c}}$, (where $T_{\mathrm{c}}$ is the Curie temperature) we extend the procedures in our recent work [16-21]. First the Holstein-Primakoff representation [3] is used to transform the Hamiltonian in the absence of pumping from components of the spin operators $\boldsymbol{S}_{i n}$ to boson creation and annihilation operators $\alpha_{i n}^{\dagger}$ and $\alpha_{i n}$. If the static applied field is along the defined $z$ direction for either the film or the wire, then $z$ is also along the preferred direction of static magnetization, so this transformation is straightforward. In the case of the wire, however, we will also include calculations for the case of a transverse applied field, along $x$ in Fig. 1b, where the individual spins are canted away from the $z$ axis and the static magnetization is spatially nonuniform. Then the procedure involves determining the equilibrium orientations of the spins for any value of the transverse field and applying the Holstein-Primakoff transformation relative to these local axes for each spin (see [21]). In both of the above cases we expand the Hamiltonian of the system for $T \ll T_{\mathrm{c}}$, as $H=H^{(2)}+H^{(3)}+H^{(4)}+\ldots$, apart from a constant, with $H^{(m)}$ denoting the term with a product of $m$ boson operators.

A canonical (generalized Bogoliubov) transformation can next be introduced, following [18, 21], to diagonalize $H^{(2)}$ giving

$$
H^{(2)}=\sum_{\boldsymbol{q}, l} \omega_{l}(\boldsymbol{q}) \alpha_{q, l}^{\dagger} \alpha_{q, l},
$$

where $\omega_{l}(\boldsymbol{q})$ is the frequency of the non-interacting SW branch $l(l=1,2, \ldots, N)$ at wave vector $\boldsymbol{q}$. Here $\boldsymbol{q}$ is a two-dimensional (2D) wave vector in the case of a film, with $\boldsymbol{q}=\left(q_{x}, q_{z}\right)$ and a $1 \mathrm{D}$ wave vector for a nanowire, with $\boldsymbol{q}=\left(q_{z}\right)$. In the case of parallel pumping, where the microwave field $\boldsymbol{h}_{0}$ in Eq. (3) is parallel to the magnetization direction, the higher-order $H^{(3)}$ and $H^{(4)}$ expansion terms, which describe three-magnon and fourmagnon processes respectively, are unimportant. This is not the case, however, for perpendicular pumping.

For simplicity, we start with parallel pumping. The additional Hamiltonian term that describes the SW instability in this case is

$$
\begin{aligned}
& H_{\mathrm{p}}^{(2)}=-g \mu_{\mathrm{B}} \exp \left(-\mathrm{i} \omega_{\mathrm{p}} t\right) \sum_{\boldsymbol{q}, l, l^{\prime}} P_{l, l^{\prime}}(\boldsymbol{q}) \alpha_{q, l^{\dagger}}^{\dagger} \alpha_{-q, l^{\prime}}^{\dagger} \\
& \quad+\text { H.c. }
\end{aligned}
$$

The amplitude factor $P_{l, l^{\prime}}(\boldsymbol{q})$ is quoted in [16] for the film case, and for the present calculations we have derived analogous expressions for the wire geometry that apply when the static external field of magnitude $H_{0}$ is along either the $z$ axis (longitudinal field) or the $x$ axis (transverse field).

The next step is to derive the rate equations for the boson operators $\alpha_{q, l}^{\dagger}$ and $\alpha_{-q, l}$ by forming their commutator with the effective Hamiltonian $H_{\mathrm{eff}}=H^{(2)}+H_{\mathrm{p}}^{(2)}$ for parallel pumping. This eventually gives rise to a set of $N$ coupled equations for the boson operators $\alpha_{q, l}$, and another $N$ equations for their conjugates, in the form 


$$
\begin{aligned}
& \frac{\mathrm{d}}{\mathrm{d} t} \alpha_{q, l}=-\left\{\mathrm{i} \omega_{l}(\boldsymbol{q})+\eta_{l}(\boldsymbol{q})\right\} \alpha_{\boldsymbol{q}, l} \\
& \quad-\mathrm{i} h_{0} \exp \left(-\mathrm{i} \omega_{\mathrm{p}} t\right) \sum_{l^{\prime}} P_{l, l^{\prime}}(\boldsymbol{q}) \alpha_{-\boldsymbol{q}, l^{\prime}}^{\dagger}
\end{aligned}
$$

Here we have introduced the role of energy dissipation phenomenologically via the damping constant $\eta_{l}(\boldsymbol{q})$ for the SW branch $l$, following the approach commonly used in macroscopic theories (see, e.g., [9-13]) as well as in [16] for a microscopic approach.

A numerical procedure to solve for the instability thresholds arising from the above type of coupled equations is outlined in $[13,14,16]$. In general, when the driving field $h_{0}$ is larger than a threshold value $h_{\mathrm{c}}$, the rate at which energy is pumped into the SW system exceeds that lost to the system through relaxation mechanisms. The SW population increases exponentially above this threshold until it reaches a saturation depending on the nonlinear interactions. The set of coupled rate equations can be recast into a matrix form as an eigenvalue problem, and the onset of instability is associated with a change of sign of one of the eigenvalues. Typically, the calculations are carried out numerically as described later, but in a few special cases there are analytic expressions. For example, in the special case of a single layer film $(N=1)$, where there is only one SW branch $(l=1)$, we find analytically that

$$
h_{\mathrm{c}}=\frac{\sqrt{\left(\eta_{1}(\boldsymbol{q})\right)^{2}+\left(\Delta \omega_{1}(\boldsymbol{q})\right)^{2}}}{\left|P_{1,1}(\boldsymbol{q})\right|},
$$

where $\Delta \omega_{1}(\boldsymbol{q})=\omega_{1}(\boldsymbol{q})-\frac{1}{2} \omega_{\mathrm{p}}$. For a nanowire in the simplest non-trivial case, which corresponds to $2 \times 1(N=2)$, we find that $h_{\mathrm{c}}=\min \left(h_{\mathrm{c} 1}, h_{\mathrm{c} 2}\right)$ for the two $\mathrm{SW}$ branches, where

$$
h_{\mathrm{c} i}=\frac{\sqrt{\eta_{1}(\boldsymbol{q}) \eta_{2}(\boldsymbol{q})+\left(\Delta \omega_{i}(\boldsymbol{q})\right)^{2}}}{\left|P_{i, i}(\boldsymbol{q})\right|}, \quad(i=1,2) .
$$

In order to study SW instability thresholds under condition of perpendicular pumping an analogous approach can be followed. For simplicity, we shall restrict the discussion to the film case, where perpendicular pumping means that the microwave field $\boldsymbol{h}_{0}$ can be considered in terms of components along the in-plane $x$ direction or the out-of-plane $y$ direction. We conclude later that the corresponding threshold fields, denoted as $h_{c x}$ and $h_{c y}$, are quite different. The effective Hamiltonian that leads to the instabilities of the system for the first-order Suhl process $[4,5]$ is $H_{\text {eff }}=H^{(2)}+H^{(3)}+H_{\mathrm{p}}^{\perp}$, where the threemagnon term now plays an important role, along with the perpendicular microwave pumping term found explicitly from Eq. (3) and use of the Holstein-Primakoff transformation. The relevant part of $H^{(3)}$ that drives the SW instability consists of terms of the form $\alpha_{q, l}^{\dagger} \alpha_{-q, l^{\prime}}^{\dagger} \alpha_{0, l^{\prime \prime}}$ for the operators, where the uniform (or zero wave-vector) mode can be replaced by a resonance term, as in [10, 11]. The second-order Suhl process, which depends on the four-magnon term $H^{(4)}$, will not be considered here.

Then, following the approach as described earlier, the set of $N$ coupled rate equations can be formed. We eventually find results that are formally similar to Eq. (6), but with $P_{l, l^{\prime}}(\boldsymbol{q})$ replaced by different expressions. Numerical examples will be given in Sect. 3; details of the calculations will be presented elsewhere.

\section{Numerical applications}

In this section the previous theory will be applied to study the SW instabilities for some ultrathin films and nanowires. Examples for microwave pumping in either the parallel or perpendicular configuration will be given for the dispersion relations of the quantized SW modes and their instability thresholds in different structures and magnetic materials. Typically we consider films with values of $N$ up to about 50, and wires with different crosssectional aspect ratios $N_{x} / N_{y}<1$ and $N=N_{x} N_{y}$ up to about 15 . The SWs in films, which are characterized by a $2 \mathrm{D}$ wave vector, depend on the magnitude $q=|\boldsymbol{q}|$ and inplane propagation angle $\varphi=\tan ^{-1}\left(q_{x} / q_{z}\right)$. Hence they occur in bands that may become overlapping at large $N$. On the other hand, the SWs in wires depend only on a $1 \mathrm{D}$ wave vector so the spectrum consists of distinct lines (branches) that sometimes may hybridize.

Numerical calculations will be presented using bulk parameters appropriate to four magnetic materials, which are representative of widely differing dipolar-to-exchange interaction ratios. Specifically, the materials are YIG (as a strong-exchange case), EuO and $\mathrm{EuS}$ (as intermediate cases), and $\mathrm{GdCl}_{3}$ (as a strongly-dipolar case). For YIG we have approximately $H_{\mathrm{ex}}=6 S J / g \mu_{\mathrm{B}}=200 \mathrm{~T}$ for the exchange field, $M_{\mathrm{s}}=g \mu_{\mathrm{B}} S / a^{3}=0.176 / 4 \pi \mathrm{T}$ for the saturation magnetization, $S=5 / 2$, and $g \mu_{\mathrm{B}}=$ $28.0 \mathrm{GHz} / \mathrm{T}[16]$. Likewise, for $\mathrm{EuO}$ we take $H_{\mathrm{ex}}=38 \mathrm{~T}$, $4 \pi M_{\mathrm{s}}=2.4 \mathrm{~T}, S=7 / 2$, and $g \mu_{\mathrm{B}}=28.0 \mathrm{GHz} / \mathrm{T}$; for EuS we take $H_{\mathrm{ex}}=9.4 \mathrm{~T}, 4 \pi M_{\mathrm{s}}=1.5 \mathrm{~T}, S=7 / 2$, and $g \mu_{\mathrm{B}}=28.0 \mathrm{GHz} / \mathrm{T}$; and finally for $\mathrm{GdCl}_{3}$ we assume $H_{\mathrm{ex}}=0.54 \mathrm{~T}, 4 \pi M_{\mathrm{s}}=0.82 \mathrm{~T}, S=7 / 2$, and $g \mu_{\mathrm{B}}=28.0 \mathrm{GHz} / \mathrm{T}$. Another parameter required for the calculations is the damping $\eta_{l}(\boldsymbol{q})$, which in principle may depend on $\boldsymbol{q}$ and on the branch label $l$. Here we will follow the simplification commonly adopted in the cited macroscopic calculations of adopting a constant value. YIG has a very small damping, for which we may assume a typical experimental value of $0.001 \mathrm{GHz}$ (or $\Delta H \approx 0.3$ Oe for the equivalent resonance half-linewidth given by $\left.\eta / g \mu_{\mathrm{B}}\right)$. For damping of the other materials we consider several values between 0.01 and $0.1 \mathrm{GHz}$. More discussion of parameter values is given in [16], and references therein.

We start with the applications to films. In Fig. 2 the lowest SW frequencies are plotted as a function of $q a$ for several values of the propagation angle $\varphi$ for (a) a EuO film with $N=12$ and (b) a YIG film with $N=50$. For any nonzero $q a$ there is a range of frequencies, leading to SW bands (with $\varphi=0$ and $\pi / 2$ defining the lower and upper boundaries, respectively). The widths of these bands become zero in the limit of $q a \rightarrow 0$, leading to a series of discrete frequencies, of which the lowest lies very close to the angular frequency $g \mu_{\mathrm{B}} \sqrt{H_{0}\left(H_{0}+4 \pi M_{\mathrm{s}}\right)}$ of the 

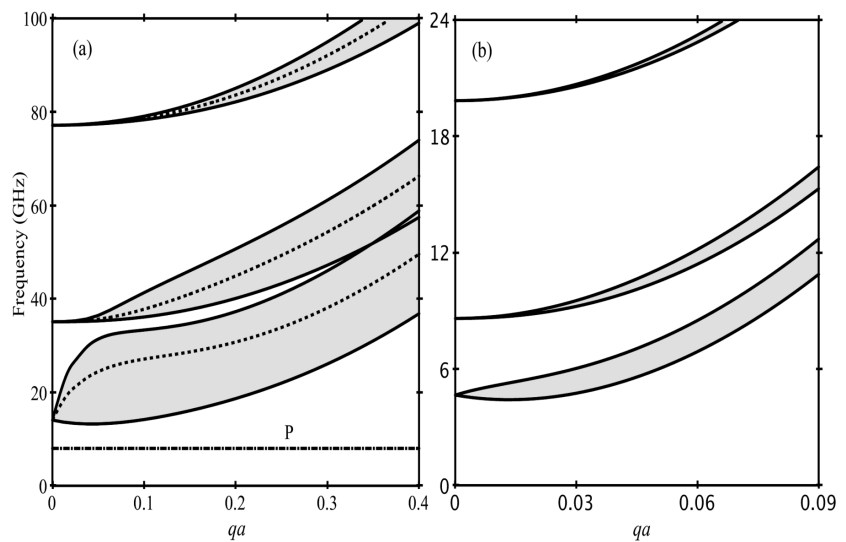

Fig. 2. SW frequencies for the lowest three bands (shaded areas) in ultrathin films plotted versus dimensionless in-plane wave vector $q a$ for (a) $\mathrm{EuO}$ with $N=$ 12 and (b) YIG with $N=50$, with $H_{0}=0.1 \mathrm{~T}$ in both cases. The bottom and top of each band corresponds to propagation angle $\varphi=0$ and $\pi / 2$, respectively. Also in case (a) the line for $\varphi=\pi / 4$ is included (dashed line) and the horizontal line (labeled $\mathrm{P}$ ) indicates one half of the pumping frequency to be considered.

Damon-Eshbach (DE) mode at $q=0$ in magnetostatic theory [24]. This expression yields 14.0 and $4.65 \mathrm{GHz}$ using the quoted $M_{\mathrm{s}}$ and $H_{0}$ for Fig. 2a and b, respectively, which are seen to be close to the numerical calculations. The separation of the bands increases with $H_{\mathrm{ex}}$ and decreases with $N$. This means relatively narrow, separated bands for YIG and wider bands for EuO that are beginning to overlap for $N=12$ in Fig. 2(a). The top of the DE mode in magnetostatic theory corresponds to $g \mu_{\mathrm{B}}\left(H_{0}+2 \pi M_{\mathrm{s}}\right)$. This yields $36.4 \mathrm{GHz}$ for $\mathrm{EuO}$ in Fig. 2a, which is roughly where the first band levels off at small $q a \approx 0.1$ before increasing further due to exchange effects at larger wave vectors. The corresponding value is $5.3 \mathrm{GHz}$ for the YIG film in Fig. 2b. Another notable feature is the initial dip of the lower $(\varphi=0)$ boundary of the first band, which is expected by analogy with properties of the magnetostatic backward volume modes [24]. Eventually at larger $q a$ this boundary bends upwards due to effects of exchange interactions, which are stronger in YIG.

In Figs. 3 and 4 we present calculations for the threshold field $h_{\mathrm{c}}$ (relative to $\Delta H$ ) plotted versus applied field $H_{0}$ for $\mathrm{EuO}$ films when the pumping-field frequency $\omega_{\mathrm{p}} / 2 \pi=16 \mathrm{GHz}$. These results are the analogs of the so-called "butterfly curves" in macroscopic samples. The parallel pumping case is considered in Fig. 3 where we compare our results for three values of $N=3,12$ and 36 . The three curves have a similar form with a mainly flat region below a cusp field, whereas above this field (occurring at about $0.07,0.08$, and $0.16 \mathrm{~T}$, respectively, for the three values of $N$ ) the threshold $h_{\mathrm{c}}$ increases sharply. This behaviour can be understood on the basis that the decay instability is generally dominated by the formation of two degenerate SWs with $\omega_{i}(\boldsymbol{q}) \approx \omega_{\mathrm{p}} / 2$ (see, e.g. [111] and Eq. (7) for a special case). The horizontal line

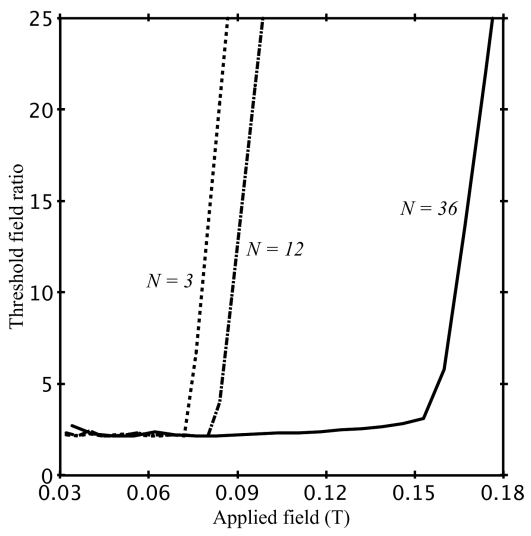

Fig. 3. SW instability thresholds $h_{\mathrm{c}}$ expressed as a ratio of the half-linewidth $\Delta H$ and plotted versus applied field $H_{0}$ for $\mathrm{EuO}$ films with $N=3,12$ and 36. Parallel pumping at frequency $\omega_{\mathrm{p}} / 2 \pi=16 \mathrm{GHz}$ is considered with $\eta=0.1 \mathrm{GHz}$.

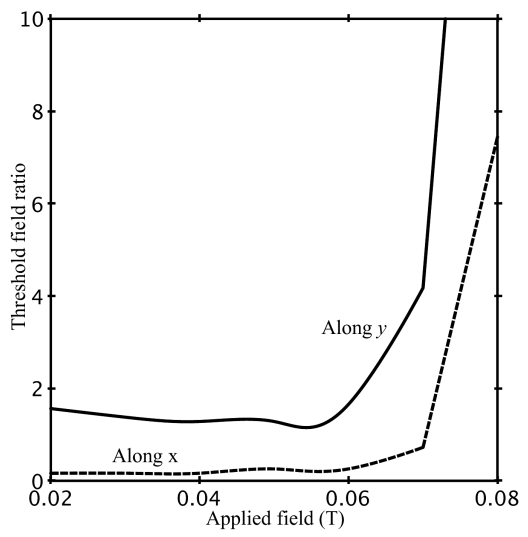

Fig. 4. SW instability thresholds $h_{\mathrm{c}}$ expressed as a ratio of the half-linewidth $\Delta H$ and plotted versus applied field $H_{0}$ for a EuO film with $N=10$ and $\eta=0.01 \mathrm{GHz}$. Perpendicular pumping at $\omega_{\mathrm{p}} / 2 \pi=16 \mathrm{GHz}$ is considered for the microwave field along $x$ (dashed line) and along $y$ (solid line).

$\mathrm{P}$ drawn at $8 \mathrm{GHz}$ in the dispersion curves of Fig. 2a when $H_{0}=0.1 \mathrm{~T}$ lies below the bottom of the first SW band, so the decay threshold is high, whereas at smaller $H_{0}$ this line would intersect one or more of the SW bands and the decay would be more likely. The results shown in Fig. 4 apply for the first-order Suhl effect in the case of perpendicular pumping. The two curves, which have a similar overall form to those in Fig. 3, show the instability threshold curves for the cases where the pumping field is along $x$ (parallel to the film surfaces) or along $y$ (perpendicular to the film surfaces). We find that the $h_{\mathrm{c}}$ values are larger in the latter case, and this is related to the ellipticity of the spin precession.

Next we consider examples for nanowires. In Fig. 5 the frequencies of the lowest SW branches of a $4 \times 3$ wire in a longitudinal applied field are plotted as a function of $q a$ using parameters for $\mathrm{GdCl}_{3}$, which is a strongly-dipolar material as mentioned. Since we now have a 1D wave 


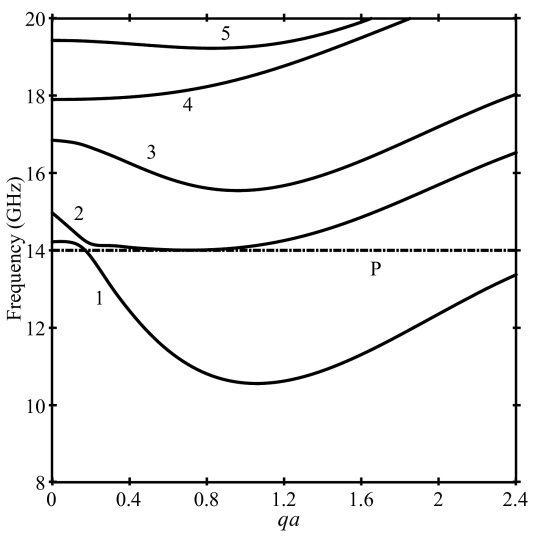

Fig. 5. SW frequencies plotted versus dimensionless longitudinal wave vector $q a$ for the lowest five branches of a $4 \times 3$ nanowire $(N=12)$ taking parameters as for $\mathrm{GdCl}_{3}$ and a longitudinal applied field $H_{0}=0.117 \mathrm{~T}$ and $\eta=0.1 \mathrm{GHz}$. The horizontal line (labeled $\mathrm{P}$ ) indicates one half of the pumping frequency to be considered

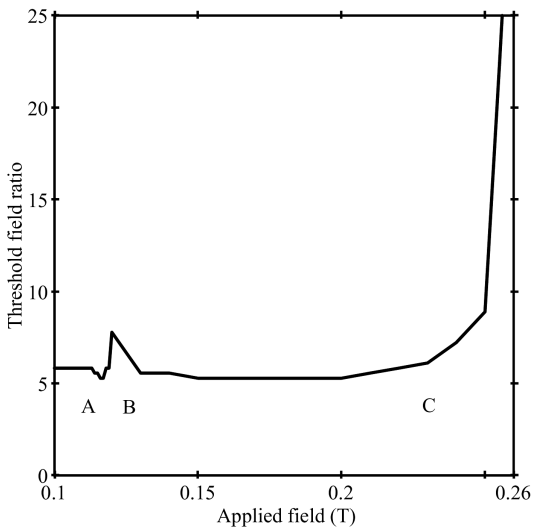

Fig. 6. SW instability threshold $h_{\mathrm{c}}$ expressed as a ratio of the half-linewidth $\Delta H$ and plotted versus applied field $H_{0}$ for a $4 \times 3(N=12) \mathrm{GdCl}_{3}$ nanowire. Parallel pumping at $\omega_{\mathrm{p}} / 2 \pi=28 \mathrm{GHz}$ is considered with $\eta=0.1 \mathrm{GHz}$.

vector (by contrast with the film case), the spectrum consists of a series of curves for the different branches instead of bands. Some branches show a minimum at nonzero wave-vector, which is a result of the interplay between the dipolar and exchange interactions (with features analogous to the magnetostatic backward volume modes characteristic of the wave vector being parallel to the net magnetization). In addition, we see some effects of hybridization (with mode repulsion) between branches labelled 1 and 2 for small $q a$. The butterfly curve for the same nanowire with parallel pumping is given in Fig. 6 , which has several features labelled as $\mathrm{A}$ (at $H_{0}=0.11 \mathrm{~T}$ ), $\mathrm{B}\left(\right.$ at $\left.H_{0}=0.117 \mathrm{~T}\right)$ and $\mathrm{C}\left(\right.$ at $\left.H_{0}=0.23 \mathrm{~T}\right)$. They can all be associated with $H_{0}$ values at which the line $\mathrm{P}$ in Fig. 5, drawn at one-half of the pumping frequency, coincides with a specific feature of the SW dispersion. Point A corresponds to the field when the one-half frequency is coincident with the $q a \approx 0$ part of branch 1 , point $\mathrm{B}$

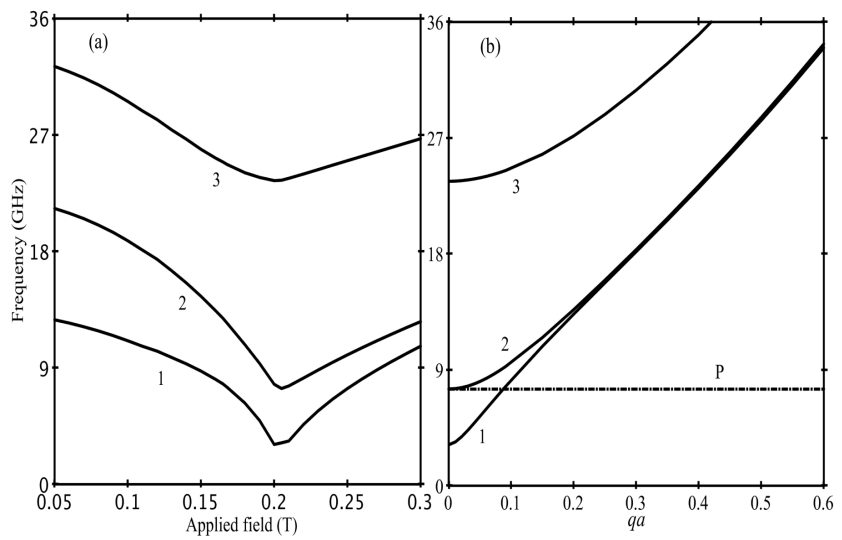

Fig. 7. SW frequencies for the lowest three branches of a $12 \times 1 \mathrm{EuS}$ nanowire $(N=12)$ in a transverse applied field with $\eta=0.03 \mathrm{GHz}$ : (a) plotted versus $H_{0}$ below and above the transition at $0.20 \mathrm{~T}$ for a fixed $q a \approx 0$; (b) plotted versus $q a$ for a fixed field $H_{0}=0.21 \mathrm{~T}$ which is above the transition. The horizontal line (labeled $\mathrm{P}$ ) in (b) indicates one half of the pumping frequency to be considered.

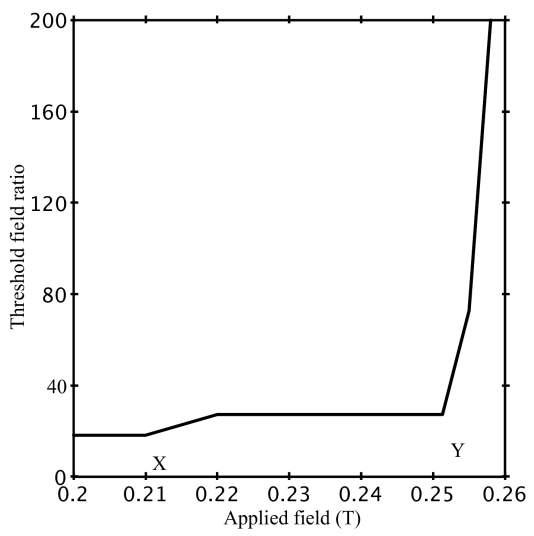

Fig. 8. SW instability threshold $h_{\mathrm{c}}$ expressed as a ratio of the half-linewidth $\Delta H$ and plotted versus the transverse applied field $H_{0}$ (above the transition field in Fig. 7) for a $12 \times 1(N=12) \mathrm{EuS}$ nanowire with $\eta=0.03 \mathrm{GHz}$. Parallel pumping at $\omega_{\mathrm{p}} / 2 \pi=15 \mathrm{GHz}$ is considered.

corresponds to the field value in Fig. 5 where the line is just touching the minimum in branch 2 , and point $\mathrm{C}$ is analogous to the main cusp point in previous examples (i.e., the field value where the line is just touching the minimum in branch 1 ).

In Figs. 7 and 8 we examine the behaviour in a nanowire when the static field $H_{0}$ is applied transversely (in the $x$ direction), causing the spins to cant in that direction. We employ parameters for a $12 \times 1 \mathrm{EuS}$ wire (or ribbon-like structure). Results for the lowest SW frequencies plotted versus the transverse field at a fixed $q a \approx 0$ are given in Fig. 7a, where the main dip at $H_{0} \approx 0.20 \mathrm{~T}$ provides us with the critical field value above which the net spin orientation or sample magnetization is along $x$. Below the critical field the competition between the applied field and the dipolar field leads to 
SW curves that decrease gradually with increasing $H_{0}$. Above the critical field the spins are reoriented perpendicular to the wire axes and the frequencies increase with increasing $H_{0}$. Next, in Fig. $7 \mathrm{~b}$ we show the lowest SW frequencies plotted versus $q a$ at a fixed $H_{0}$ above the critical value. In this case with the longitudinal wave vector perpendicular to the net magnetization direction, the SW frequencies all increase monotonically with increasing $q a$, unlike the behaviour in a longitudinal field (see Fig. 5). The lowest two branches are well separated at small $q a$, but become approximately degenerate at larger $q a$ when exchange effects start to play a dominant role.

The corresponding butterfly curve for the transverselymagnetized EuS wire in the case of parallel pumping is given in Fig. 8. We note two features labelled $\mathrm{X}$ and $\mathrm{Y}$ at $H_{0}=0.21 \mathrm{~T}$ and $H_{0}=0.251 \mathrm{~T}$ respectively, which correspond to the one-half pumping frequency line coinciding with the minimum frequency for SW branch 2 (as in Fig. 7b) and then for SW branch 1.

\section{Conclusions}

We have presented a microscopic dipole-exchange theory for the SW instability thresholds of ferromagnetic nanostructures, specifically for films magnetized in-plane, longitudinally magnetized wires (or stripes), and transversely magnetized wires. Both the parallel and perpendicular (first-order Suhl) configurations for the pumping field were considered and the results were interpreted in terms of the SW modes, which are quantized differently in the two structures due to their differing symmetries. The results depend sensitively on the dipolar-to-exchange ratio, and this was illustrated taking parameters for different magnetic materials. In future work it would be of interest to explore more fully the role of damping and also to include effects of surface anisotropy. A complication in the case of the damping is that the spin relaxation might take place through different mechanisms (e.g., magnonmagnon interactions, scattering from impurities or surface inhomogeneities, etc.). For example, recent experiments [25] on Permalloy nanowires (stripes) showed contributions to the damping due to three-magnon processes and edge roughness.

\section{Acknowledgments}

The authors acknowledge support from the Natural Sciences and Engineering Research Council (NSERC) of Canada, as well as Compute Canada SHARCNet network.

\section{References}

[1] G.A. Melkov, Yu.V. Koblyanskiy, R.A. Slipets, A.V. Talalaevskij, A.N. Slavin, Phys. Rev. B 79, 134411 (2009).

[2] H. Ulrichs, V.E. Demidov, S.O. Demokritov, S. Urazhdin, Phys. Rev. B 84, 094401 (2011).
[3] M. Weiler, G. Woltersdorf, M. Althammer, H. Huebl, S.T.B. Goennenwein, in: Recent Advances in Magnetic Insulators - From Spintronics to Microwave Applications, Eds. M. Wu, A. Hoffmann, Elsevier, Amsterdam 2013, p. 123

[4] H. Suhl, J. Phys. Chem. Solids 1, 209 (1957).

[5] E. Schlomann, J.J. Green, U. Milano, J. Appl. Phys. 31, S386 (1960).

[6] Nonlinear Phenomena and Chaos in Magnetic Materials, Ed. P. Wigen, World Scientific, Singapore 1994.

[7] V. S. L'vov, Wave Turbulence under Parametric Excitation: Application to Magnets, Springer, Heidelberg 1994.

[8] M.G. Cottam, A.N. Slavin, in: Linear and Nonlinear Spin Waves in Magnetic Films and Superlattices, Ed. M.G. Cottam, World Scientific, Singapore 1994, p. 1.

[9] S.M. Rezende, A. Azevedo, F.M. de Aguiar, in: Linear and Nonlinear Spin Waves in Magnetic Films and Superlattices, Ed. M.G. Cottam, World Scientific, Singapore 1994, p. 335.

[10] A.G. Gurevich, G.A. Melkov, Magnetization Oscillations and Waves, CRC Press, Boca Raton 1996.

[11] D.D. Stancil, A. Prabhakar, Spin Waves: Theory and Applications, Springer, Heidelberg 2009.

[12] D.N. Chartoryzhskii, B.A. Kalinikos, O.G. Vendik, Solid State Commun. 20, 985 (1976).

[13] G. Wiese, P. Kabos, C.E. Patton, J. Appl. Phys. 74 , 1218 (1993).

[14] G. Wiese, P. Kabos, C.E. Patton, Phys. Rev. B 51, 15085 (1995).

[15] V.B. Cherepanov, A.N. Slavin, in: High Frequency Processes in Magnetic Materials, Eds. G. Srinivasan, A.N. Slavin, World Scientific, Singapore 1995, p. 250.

[16] H.T. Nguyen, M.G. Cottam, Phys. Rev. B 89, 144424 (2014).

[17] R.N. Costa Filho, M.G. Cottam, G.A. Farias, Phys. Rev. B 62, 6545 (2000).

[18] H.T. Nguyen, M.G. Cottam, J. Phys. C Condens. Matter 23, 126004 (2011).

[19] T.M. Nguyen, M.G. Cottam, Phys. Rev. B 71, 094406 (2005).

[20] H.T. Nguyen, T.M. Nguyen, M.G. Cottam, Phys. Rev. B 76, 134413 (2007).

[21] H.T. Nguyen, A. Akbari-Sharbaf, M.G. Cottam, Phys. Rev. B 83, 214423 (2011).

[22] H.T. Nguyen, M.G. Cottam, J. Phys. D Appl. Phys. 44, 315001 (2011).

[23] T. Holstein, H. Primakoff, Phys. Rev. 58, 1098 (1940).

[24] R.W. Damon, J.R. Eshbach, J. Phys. Chem. Solids 19, 308 (1961).

[25] C.T. Boone, J.A. Katine, J.R. Childress, V. Tiberkevich, A. Slavin, J. Zhu, X. Cheng, I.N. Krivorotov, Phys. Rev Lett. 103, 167601 (2009). 